\begin{tabular}{|c|c|}
\hline Title & $\begin{array}{l}\text { Feasibility and efficacy of induction docetaxel, cisplatin, and } 5 \text {-fluorouracil chemotherapy combined with concurrent } \\
\text { weekly cisplatin chemoradiotherapy for locally advanced head and neck squamous cell carcinoma }\end{array}$ \\
\hline Author(s) & $\begin{array}{l}\text { Mizumachi, T akatsugu; Homma, A kihiro; Kakizaki, Tomohiko; Sakashita, Tomohiro; Kano, Satoshi; Hatakeyama, } \\
\text { Hiromitsu; T suchiya, Kazuhiko; Y asuda, Koichi; Onimaru, Rikiya; Shirato, Hiroki; T aguchi, Jun; Shimizu, Y asushi; } \\
\text { Kinoshita, Ichiro; A kita, Hirotoshi; Fukuda, Satoshi }\end{array}$ \\
\hline Citation & $\begin{array}{l}\text { International journal of clinical oncology, 20(3), 431-437 } \\
\text { https://doi.org/10.1007/s10147-014-0726-y }\end{array}$ \\
\hline Issue Date & 2015-06 \\
\hline Doc URL & http:/hdl.handle.net/2115/61985 \\
\hline Rights & The final publication is available at Springer via http://dx.doi.org/10.1007/s10147-014-0726-y \\
\hline Type & article (author version) \\
\hline File Information & manuscript.pdf \\
\hline
\end{tabular}

Instructions for use 
Title: Feasibility and efficacy of induction docetaxel, cisplatin, and 5-fluorouracil chemotherapy combined with concurrent weekly cisplatin chemoradiotherapy for locally advanced head and neck squamous cell carcinoma

Takatsugu Mizumachi ${ }^{1}$, Akihiro Homma ${ }^{1}$, Tomohiko Kakizaki ${ }^{1}$, Tomohiro Sakashita ${ }^{1}$, Satoshi Kano ${ }^{1}$, Hiromitsu Hatakeyama ${ }^{1}$, Kazuhiko Tsuchiya ${ }^{2}$, Koichi Yasuda ${ }^{2}$, Rikiya Onimaru $^{2}$, Hiroki Shirato ${ }^{2}$, Jun Taguchi ${ }^{3}$, Yasushi Shimizu ${ }^{3}$, Ichiro Kinoshita ${ }^{3}$, Hirotoshi Akita $^{3}$, Satoshi Fukuda ${ }^{1}$

${ }^{1}$ Department of Otolaryngology - Head and Neck Surgery, Hokkaido University Graduate School of Medicine

${ }^{2}$ Department of Radiation Medicine, Hokkaido University Graduate School of Medicine

${ }^{3}$ Department of Medical Oncology, Hokkaido University Graduate School of Medicine

Corresponding author: Takatsugu Mizumachi

Mailing address: Department of Otolaryngology- Head and Neck Surgery, Hokkaido University Graduate School of Medicine, Kita 15, Nishi 7, Kita-ku, Sapporo 060-8638, Japan

Phone: $+81-11-706-5958$

Fax: $+81-11-717-7566$

E-mail: mizumati@med.hokudai.ac.jp 


\begin{abstract}
Background: To evaluate the feasibility of induction docetaxel, cisplatin, and 5-fluorouracil chemotherapy followed by concurrent weekly cisplatin chemoradiotherapy for patients with locally advanced head and neck squamous cell carcinoma (HNSCC).
\end{abstract}

Methods: Between $\underline{\mathbf{2 0 1 0}}$ and 2013, 30 patients with Stage IV HNSCC were treated in Hokkaido University Hospital with three cycles of induction chemotherapy (docetaxel $75 \mathrm{mg} / \mathrm{m}^{2}$ and cisplatin $75 \mathrm{mg} / \mathrm{m}^{2}$, day 1 ; and 5 -fluorouracil $750 \mathrm{mg} / \mathrm{m}^{2} /$ day $120 \mathrm{~h}$ continuous infusion, every 3 weeks) followed by concurrent weekly cisplatin $\left(40 \mathrm{mg} / \mathrm{m}^{2}\right.$, on weeks 1, 2, 3, 5, 6 and 7) chemoradiotherapy.

Results: Three courses of induction chemotherapy were performed in 25 patients (83\%), with grade 3-4 toxicities during induction chemotherapy observed in 22 patients (73\%). The major toxicities were hematologic, with 22 cases (73\%) showing grade 3-4 neutropenia. Radiotherapy was completed (70Gy) in 29 patients (97\%), while a total of 19 patients (63\%) completed five (13 patients) or six (6 patients) courses of the chemotherapy. During concurrent chemoradiotherapy, no grade 4 hematological toxicities were observed. Grade 4 dermatitis was observed in one patient, and grade 3 mucositis was observed in 12 patients. There were no treatment-related deaths during 
the induction chemotherapy or concurrent chemoradiotherapy. The 1- and 2-year progression-free survival and 1- and 2-year overall survival rates were $86 \%, 72 \%$, and $89 \%, 81 \%$, respectively.

Conclusion: Sequential therapy composed of induction chemotherapy followed by concurrent weekly cisplatin chemoradiotherapy is feasible, showing encouraging results in patients with locally advanced HNSCC. Concurrent weekly cisplatin chemoradiotherapy following induction chemotherapy appears to be a suitable alternative to three-weekly high-dose cisplatin therapy. 


\section{Mini Abstract}

Sequential therapy composed of induction chemotherapy followed by concurrent weekly cisplatin chemoradiotherapy is feasible for and shows encouraging results in patients with locally advanced HNSCC.

Key Words: head and neck cancer, induction chemotherapy, chemoradiotherapy 


\section{Introduction}

For the treatment of patients with locally advanced head and neck squamous cell carcinoma (HNSCC), concurrent chemoradiotherapy has been demonstrated to benefit survival in many clinical trials and the meta-analysis of chemotherapy in head and neck cancer (MAHC-NC) [1]. Concurrent chemoradiotherapy is applied to both patients with unresectable disease and patients with resectable disease who refuse radical surgery. Three-weekly cisplatin at a dose of $100 \mathrm{mg} / \mathrm{m}^{2}$ is considered to be the standard treatment for patients with advanced HNSCC $[2,3]$. However, this protocol has been reported to be associated with severe acute and late toxicities. Alternative cisplatin dosing schedules are sometimes used due to improved patient tolerance [4-6]. We previously reported that weekly cisplatin at a dose of $40 \mathrm{mg} / \mathrm{m}^{2}$ was easier to manage than three-weekly cisplatin [7]. Although concurrent chemoradiotherapy reduces the rates of locoregional failure, distant metastases represent a major cause of treatment failure in the patients treated by concurrent chemoradiotherapy [8].

Induction chemotherapy has been added to chemoradiotherapy in an attempt to decrease the likelihood of distant metastasis, improve local regional control, and aid organ preservation [9]. Two Phase III trials that focused on the efficacy of induction chemotherapy with docetaxel, cisplatin, and 5-fluorouracil (5-FU) (DCF) have shown 
promising results $[10,11]$. Cisplatin, carboplatin, docetaxel, cisplatin plus 5-FU, and cetuximab regimens have been reportedly used in concurrent chemoradiotherapy following induction chemotherapy $[9,10,12,13]$. In our study, sequential therapy, consisting of induction chemotherapy involving three cycles of docetaxel and cisplatin plus 5-FU, followed by concurrent chemoradiotherapy with six cycles of weekly cisplatin and radiotherapy was administrated.

The objectives of this study were to evaluate the compliance, response rate and toxicities of the sequential therapy for patients with locally advanced HNSCC.

\section{PATIENTS AND METHODS}

\section{Patients}

We retrospectively identified 30 consecutive patients with previously untreated stage IV, M0 unresectable HNSCC of the oropharynx, hypopharynx, or larynx treated with curative intent at Hokkaido University by a regimen of induction chemotherapy followed by concurrent chemoradiotherapy between 2010 and 2012. The criteria for inoperability were technical unresectability, low surgical curability, and organ preservation. All patients were 70 years old or younger and had not received any previous treatment for the tumor. Patients were required to be free of other active 
cancers as well as distant metastases. An Eastern Cooperable Oncology Group performance status of $0-1$ was required in addition to the following criteria: a white cell count of at least $4,000 / \mathrm{mm}^{3}$, a platelet count of at least $100,000 / \mathrm{mm}^{3}$, a hemoglobin concentration of at least $9.5 \mathrm{~g} / \mathrm{dL}$, serum glutamic oxaloacetic transaminase and serum glutamic pyruvic transaminase levels of less than twice the upper limit of the normal range, a total bilirubin concentration of less than $2.0 \mathrm{mg} / \mathrm{dL}$, a serum creatinine concentration of less than $1.5 \mathrm{~g} / \mathrm{dL}$, a blood urea nitrogen concentration of less than 25 $\mathrm{mg} / \mathrm{dL}$, and a creatinine clearance of more than $60 \mathrm{~mL} / \mathrm{min}$. The disease had to be measurable or amenable to evaluation, and had to be documented as precisely as possible before treatment by endoscopy, including computed tomography (CT) and/or magnetic resonance imaging (MRI). All patients were initially evaluated by a multidisciplinary team consisting of head and neck surgeons, radiation oncologists, and medical oncologists. The tumors were classified according to the 2002 Union Internationale Contre le Cancer (UICC) staging system.

\section{HPV typing by multiplex PCR}

The HPV infection status for patients with oropharyngeal squamous cell carcinoma (OPSCC) was analyzed using multiplex PCR, which can detect 16 HPV 
genotypes (types $6,11,16,18,30,31,33,35,39,45,51,52,56,58,59$, and 66 ), as

described previously [14].

\section{Induction Chemotherapy}

Induction chemotherapy consisted of docetaxel at $75 \mathrm{mg} / \mathrm{m}^{2}$ on day 1 , cisplatin at $75 \mathrm{mg} / \mathrm{m}^{2}$ on day 1 , and 5 -fluorouracil at a dose of $750 \mathrm{mg} / \mathrm{m}^{2}$ by 24 -hour continuous infusion for 5 days; three cycles at 21-day intervals were planned. All patients were given adequate hydration and anti-emetics (5-HT3 antagonists and dexamethasone and/or neurokinin-1 receptor antagonist).

\section{Concurrent chemotherapy}

After induction chemotherapy, concurrent chemoradiotherapy was initiated between 3-4 weeks after the last chemotherapy cycle. Weekly cisplatin was administered at a dose of $40 \mathrm{mg} / \mathrm{m}^{2}$ on weeks $1,2,3,5,6$ and 7 of the radiotherapy. Patients received prophylactic hydration and 5-HT3 antagonists plus dexamethasone and/or neurokinin-1 receptor antagonist for anti-emetic prophylaxis. The intended maximum total dose of cisplatin was $240 \mathrm{mg} / \mathrm{m}^{2}$. Preparation for percutaneous endoscopic gastrostomy feeding prior to treatment was recommended. The use of 
non-steroidal anti-inflammatory drugs was avoided in order to prevent any synergistic toxic effects with cisplatin on renal function.

\section{Radiotherapy}

A standard dose of 70 Gy was delivered in 35 daily fractions over 7 weeks to all of the patients. All of the patients received radiotherapy (40 Gy/20 fractions/4 weeks) in the form of 4 or $6 \mathrm{MV}$ photons, produced by a linear accelerator to the primary sites and regional lymphatic area. The treatment was planned using a CT simulator and a three-dimensional dose-calculation computer. For patients who were suspected of having lymph-node metastases, the lower-neck region and supraclavicular fossa were prophylactically irradiated with a total of 40 Gy using an anterior single port. Electron beams were used to boost the dose delivered to the posterior cervical lymph nodes. The dose delivered to the spinal cord was kept below 40 Gy in all instances. After the initial dose of 40 Gy had been administered, an additional dose of 30 Gy was given with a shrunken field in 15 fractions over 3 weeks.

\section{Evaluation of Toxicity and Response}


Toxicities were graded using the Common Terminology Criteria for Adverse Events (NCI-CTCAE) Version 3.0. For measurable lesions, responses were evaluated from CT scans of the neck and chest two weeks after the last course of induction chemotherapy and 6-8 weeks after radiotherapy using the Response Evaluation Criteria in Solid Tumors (RECIST).

\section{Statistical Considerations}

Data on disease site, Tumor-Node-Metastasis (TNM) stage, RT dose/fractionation and chemotherapy regimen were collected. Incidences of delays to therapy, acute toxicity, dose reduction and missed treatments for both chemotherapy and RT were also recorded.

The primary endpoint was treatment compliance. Complete treatment delivery was defined as the administration of the 70 Gy RT dose within 63 days, and the completion of five or six courses of cisplatin. Treatment compliance was evaluated based on the rate of complete treatment delivery.

Cases of persistent or recurrent primary disease after the completion of CRT were considered to be local failures, even if salvage was successful. The probabilities of 
overall survival, which included death from any cause, and the local control rates (the local progression-free rates computed from the beginning of treatment until the time of local relapse) were calculated by the Kaplan-Meier method.

\section{RESULTS}

\section{Patient Characteristics}

Of the 61 HNSCC patients who fulfilled the eligibility criteria described in the PATIENTS AND METHODS section, 30 patients (27 males and 3 females) were enrolled in the study. Table 1 shows the patient and tumor characteristics. The patients ranged in age from 40 to 69 years (median $=57$ years). The most common site of the primary disease was the oropharynx (15 patients), followed by the hypopharynx (13 patients) and larynx (2 patients). The clinical stages are listed in Table 2 . The number of the patients with Stage IVA and IVB disease was 24 and 6, respectively. The median follow-up period was 18 months (range, 5-38 months).

Of the 15 patients with OPSCC, HPV infection status was analyzed in 11. Six patients were found to be HPV-positive, with five positive for HPV16, and one positive for HPV18 (Table 3). 


\section{Induction chemotherapy}

Of the 30 patients, $25(83 \%)$ underwent three cycles of treatment at the planned doses. Three patients (10\%) received two cycles, and 2 patients $(7 \%)$ received only one cycle due to toxicity (Table 3 and 4). The median day of the administration of the second cycle of induction chemotherapy was Day 23 (range 21-30), and the median day of the administration of the third cycle of induction chemotherapy was Day 44

(range 42-58). No dose modifications were made in any patient.

The toxicity of the induction chemotherapy is summarized in Table $\underline{\mathbf{5}}$. Grade 3-4 toxicities during induction chemotherapy were recorded in 22 patients $(73 \%)$. The major toxicities were hematologic, with 22 cases (73\%) of grade 3-4 neutropenia. Grade 3 diarrhea was observed in 3 patients $(10 \%)$.

The overall response rate after induction chemotherapy was 83\% (25/30) (Table 3 and 4). Only one patient experienced tumor progression, due to the appearance of contralateral neck node metastasis, and bilateral neck dissection was required before concurrent chemoradiotherapy (Case \#16, Table 4). Another patient with stable disease with regard to lymph nodes after induction chemotherapy underwent neck dissection followed by chemoradiation as the neck disease was considered to be incurable by chemoradiation (Case \#7, Table 3). 


\section{Concurrent chemoradiotherapy}

Of the 30 patients, 29 patients received a full dose (70 Gy) of radiotherapy, with one patient receiving only 50Gy of radiotherapy after refusing to undergo further radiotherapy (Case \#6, Table 3). A total of 19 patients (63\%) completed five (13 patients) or six (6 patients) courses of the chemotherapy, with 6 patients receiving four courses, 4 patients receiving three courses, and one patient receiving just two courses of the chemotherapy. The average total dosage of cisplatin during radiotherapy was $185 \mathrm{mg} / \mathrm{m}^{2}\left(\right.$ median $\left.=200 \mathrm{mg} / \mathrm{m}^{2}\right)$ (Table 3 and 4$)$.

Acute toxicities during the concurrent chemoradiation are summarized in Table 6. No grade 4 hematological toxicities were observed. Grade 4 dermatitis was observed in one patient, and grade 3 mucositis was observed in 12 patients. There were no treatment-related deaths during the induction chemotherapy or concurrent chemoradiotherapy.

\section{Response after completion of concurrent chemoradiotherapy (Table 3 and 4)}

After completion of concurrent chemoradiotherapy, 26 patients (87\%) achieved a complete response, and only $1(3 \%)$ experienced disease progression due to lung 
metastasis (Case \#16, Table 4). Two patients who achieved PR or SD for the neck

site with CR for the primary site later underwent salvage neck dissection for residual disease after chemoradiation (Case \#5, Table 3 and Case \#26, Table 4).

\section{Outcomes}

Progression-free survival and overall survival are shown in Figure 1. The 1and 2-year progression-free survival and 1- and 2-year overall survival rates were $86 \%$, $72 \%$, and $89 \%, 81 \%$, respectively. The 1 - and 2-year progression-free survival as well as the 1- and 2- year overall survival rates were all $83.3 \%$.

Of the patients with HPV-positive OPSCC, the 1- and 2-year progression-free survival and overall survival rates were both $83.3 \%$. Of the patients with HPV-negative OPSCC, the 1- and 2-year progression-free survival and 1- and 2-year overall survival rates were $100 \%, 60 \%$, and $100 \%, 80 \%$, respectively. There were no statistically differences in survival rates between the HPV-positive and HPV-negative patients.

\section{Discussion}

This study shows that induction DCF chemotherapy followed by concurrent 
weekly cisplatin chemoradiotherapy was feasible for the treatment of and well-tolerated by patients with locally advanced HNSCC.

Eighty percent of the patients in this study completed three courses of induction DCF chemotherapy with acceptable toxicity. The most common grade 3-4 toxicity was neutropenia (73\%), with $27 \%$ experiencing febrile neutropenia during induction chemotherapy. In other studies on the use of DCF induction chemotherapy, the rate of grade 3-4 neutropenia was reported variously to be $31.5-83 \% \quad[10,11$, 15-17], and the rate of febrile neutropenia was reported to be $5.2-18 \%[10,11,15-17]$. Even though our results are at the higher end of the previously reported rates, there were no treatment-related deaths during the DCF induction chemotherapy. The overall response rate of the induction chemotherapy was $83 \%$. This response rate was similar to those of other studies on DCF induction chemotherapy.

In our study, weekly cisplatin concurrent chemoradiotherapy was administrated after induction chemotherapy. Sixty-three percent of patients received more than $200 \mathrm{mg} / \mathrm{m}^{2}$ of cisplatin after induction chemotherapy. We previously reported that $59 \%$ of patients received more than $200 \mathrm{mg} / \mathrm{m}^{2}$ of cisplatin when undergoing concurrent weekly cisplatin chemoradiotherapy [18]. These results suggest that the use of DCF induction chemotherapy does not prevent the use of concurrent weekly cisplatin 
chemotherapy.

The treatment of several series of patients with a combination of DCF induction chemotherapy and cisplatin-based concurrent chemoradiotherapy has been reported. In the TREMPLIN study [13], concurrent cisplatin $100 \mathrm{mg} / \mathrm{m}^{2} / 3$ week was administrated. Forty-two percent of the patients received three courses of cisplatin, $42 \%$ received two cycles, and 13\% received one cycle. Prestwich et al. [19] reported that concurrent cisplatin $100 \mathrm{mg} / \mathrm{m}^{2} / 3$ week was administrated after DCF induction chemotherapy. Five percent of the patients received three courses of cisplatin, $61 \%$ received two cycles, and 29\% received one cycle. They concluded that compliance over the three courses of concurrent chemotherapy was poor. Weekly cisplatin might be more easily managed than three-weekly cisplatin as patients can be monitored for toxicity more regularly, and the schedule can be changed, based on the patient's condition, before the effects become severe.

There were no treatment-related deaths during the concurrent weekly cisplatin chemoradiotherapy, and the toxicities of the concurrent weekly cisplatin chemoradiotherapy were considered acceptable. No patients were observed with grade 3 or greater renal dysfunction. Pain control was achieved by using opioids in place of NSAIDs to avoid renal dysfunction and this may have attenuated the potential for 
severe renal dysfunction. Based on a multicenter phase II study, Zenda et al. reported that an opioid-based systemic control program during chemoradiation may be helpful in improving compliance with chemoradiation [20]. Grade 3 mucositis was observed in $40 \%$ of patients during the concurrent weekly cisplatin chemoradiotherapy. Many of the patients were provided with gastrostomies before radiation, and several reports have shown that gastrostomies also improved compliance of chemoradiotherapy [ $\underline{\mathbf{2 0}}-22]$. These supportive procedures are necessary for successful concurrent cisplatin-based chemoradiotherapy.

The complete response rate after treatment was $86 \%$ in this study. In other studies using sequential therapy; i.e., DCF induction chemotherapy followed by concurrent chemoradiotherapy, the complete response rate was reported to be $50 \%$ by Paccagnella et al. [12] and 86\% by Prestwich et al. [19]. Sequential therapy, therefore, appears to result in high response rates in locally advanced HNSCC.

In this study, $50 \%$ of the patients had oropharyngeal cancer, which is similar to the rates of oropharyngeal cancer patients in other studies. HPV-positive oropharyngeal cancer has been shown to respond better to chemotherapy and radiotherapy in comparison with HPV-negative oropharyngeal cancer. We have previously reported that Japanese patients with HPV-positive OPSSC have markedly superior survival 
rates compared to those with HPV-negative OPSCC [14]. However, there were no

differences in survival rates between the HPV-positive and HPV-negative patients

in this study. We speculate that this was due to the small patient population and

short follow-up period. RTOG 1016 is now underway to determine whether treatment

can be de-intensified for HPV-positive OPSCC. However, several patients with HPV-positive OPSCC are known to develop distant metastasis. Spector et al. [23] reported that the OPSCC patients with matted nodes have a risk of distant metastasis. Although the value of induction chemotherapy prior to definitive concurrent chemoradiotherapy has not been firmly established, this sequential therapy should be considered for selected patients with locally advanced disease, including those with HPV-positive OPSCC.

In conclusion, induction docetaxel, cisplatin, and 5-fluorouracil chemotherapy combined with weekly cisplatin concurrent chemoradiotherapy is a feasible treatment for and has high rates of complete response in locally advanced HNSCC patients. While the role of sequential therapy remains inconclusive, this sequential therapy should be considered for patients with locally advanced HNSCC. 


\section{Conflict of Interest Statement}

No author has any conflict of interest.

\section{Acknowledgements}

This work was supported by a Grant-in-Aid for Young Scientists (B) from the Ministry

of Education, Culture, Sports, Science and Technology of Japan. 


\section{References}

1. Pignon JP, le Maitre A, Maillard E et al (2009) Meta-analysis of chemotherapy in head and neck cancer (MACH-NC): an update on 93 randomised trials and 17,346 patients. Radiother Oncol 92:4-14

2. Forastiere AA, Goepfert H, Maor M et al (2003) Concurrent chemotherapy and radiotherapy for organ preservation in advanced laryngeal cancer. N Engl J Med 349:2091-2098

3. Adelstein DJ (2003) An Intergroup Phase III Comparison of Standard Radiation Therapy and Two Schedules of Concurrent Chemoradiotherapy in Patients With Unresectable Squamous Cell Head and Neck Cancer. Journal of Clinical Oncology 21:92-98

4. Traynor AM, Richards GM, Hartig GK et al (2009) Comprehensive IMRT plus weekly cisplatin for advanced head and neck cancer: The University of Wisconsin experience. Head Neck 32:599-606

5. Newlin HE, Amdur RJ, Riggs CE et al (2010) Concomitant weekly cisplatin and altered fractionation radiotherapy in locally advanced head and neck cancer. Cancer 116:4533-4540

6. Sharma A, Mohanti BK, Thakar A et al (2010) Concomitant chemoradiation versus radical radiotherapy in advanced squamous cell carcinoma of oropharynx and nasopharynx using weekly cisplatin: a phase II randomized trial. Annals of Oncology 21:2272-2277

7. Homma A, Inamura N, Oridate N et al (2011) Concomitant Weekly Cisplatin and Radiotherapy for Head and Neck Cancer. Japanese Journal of Clinical Oncology 41:980-986

8. Posner M, Hershock D, Blajman C et al (2007) Cisplatin and fluorouracil alone or with docetaxel in head and neck cancer. N Engl J Med 357:1705-1715

9. Haddad R, O'Neill A, Rabinowits G et al (2013) Induction chemotherapy followed by concurrent chemoradiotherapy (sequential chemoradiotherapy) versus concurrent chemoradiotherapy alone in locally advanced head and neck cancer (PARADIGM): a randomised phase 3 trial. Lancet Oncol 14:257-264

10. Posner MR, Hershock DM, Blajman CR et al (2007) Cisplatin and fluorouracil alone or with docetaxel in head and neck cancer. N Engl J Med 357:1705-1715

11. Vermorken JB, Remenar E, van Herpen C et al (2007) Cisplatin, fluorouracil, and docetaxel in unresectable head and neck cancer. N Engl J Med 357:1695-1704

12. Paccagnella A, Ghi MG, Loreggian L et al (2010) Concomitant chemoradiotherapy versus induction docetaxel, cisplatin and 5 fluorouracil (TPF) followed by concomitant chemoradiotherapy in locally advanced head and neck cancer: a phase II randomized study. Ann Oncol 21:1515-1522

13. Lefebvre JL, Pointreau Y, Rolland F et al (2013) Induction Chemotherapy Followed by Either Chemoradiotherapy or Bioradiotherapy for Larynx Preservation: The TREMPLIN Randomized Phase II Study. Journal of Clinical Oncology 31:853-859 
14. Mizumachi T, Kano S, Sakashita $\mathrm{T}$ et al (2013) Improved survival of Japanese patients with human papillomavirus-positive oropharyngeal squamous cell carcinoma. Int J Clin Oncol 18:824-828

15. Adelstein DJ, Moon J, Hanna E et al (2009) Docetaxel, cisplatin, and fluorouracil induction chemotherapy followed by accelerated fractionation/concomitant boost radiation and concurrent cisplatin in patients with advanced squamous cell head and neck cancer: A Southwest Oncology Group phase II trial (S0216). Head Neck 32:221-228

16. Hitt R, Lopez-Pousa A, Martinez-Trufero J et al (2005) Phase III Study Comparing Cisplatin Plus Fluorouracil to Paclitaxel, Cisplatin, and Fluorouracil Induction Chemotherapy Followed by Chemoradiotherapy in Locally Advanced Head and Neck Cancer. Journal of Clinical Oncology 23:8636-8645

17. Pointreau Y, Garaud P, Chapet S et al (2009) Randomized Trial of Induction Chemotherapy With Cisplatin and 5-Fluorouracil With or Without Docetaxel for Larynx Preservation. JNCI Journal of the National Cancer Institute 101:498-506

18. Homma A, Inamura N, Oridate $\mathrm{N}$ et al (2011) Concomitant weekly cisplatin and radiotherapy for head and neck cancer. Jpn J Clin Oncol 41:980-986

19. Prestwich RJ, Oksuz DC, Dyker K et al (2011) Feasibility and efficacy of induction docetaxel, cisplatin, and 5-fluorouracil chemotherapy combined with cisplatin concurrent chemoradiotherapy for nonmetastatic Stage IV head-and-neck squamous cell carcinomas. Int J Radiat Oncol Biol Phys 81:e237-243

20. Zenda S, Matsuura K, Tachibana $H$ et al (2011) Multicenter phase II study of an opioid-based pain control program for head and neck cancer patients receiving chemoradiotherapy. Radiother Oncol 101:410-414

21. Zenda S, Onozawa Y, Tahara M et al (2007) Feasibility study of single agent Cisplatin and concurrent radiotherapy in Japanese patients with squamous cell carcinoma of the head and neck: preliminary results. Jpn J Clin Oncol 37:725-729

22. Rutter CE, Yovino S, Taylor R et al (2011) Impact of early percutaneous endoscopic gastrostomy tube placement on nutritional status and hospitalization in patients with head and neck cancer receiving definitive chemoradiation therapy. Head Neck 33:1441-1447

23. Spector ME, Gallagher KK, Light E et al (2012) Matted nodes: poor prognostic marker in oropharyngeal squamous cell carcinoma independent of HPV and EGFR status. Head Neck 34:1727-1733 


\section{Figure Legend}

Figure 1: Overall survival (OS) and progression-free survival (PFS) rates. 
Table 1 Patient Characteristics $(n=30)$

Age-yr

Median

57

Range

40-69

Gender

Male 27 / Female 3

ECOG performance status, $\mathrm{n}(\%)$

0

$2(6.7)$

1

$28(93.3)$

Site of primary tumor, n (\%)

Oropharynx

$15(50)$

Hypopharynx

$13(43.3)$

Larynx

$2(6.7)$

Overall stage of disease, $\mathrm{n}(\%)$

IVA

$24(80)$

IVB

$6(20)$ 
Table 2 T and $\mathrm{N}$ classification

\begin{tabular}{|c|c|c|c|c|c|c|c|}
\hline \multirow{2}{*}{$\begin{array}{l}\mathrm{T} \\
\text { classification }\end{array}$} & \multicolumn{7}{|c|}{$\mathrm{N}$ classification } \\
\hline & 0 & 1 & $2 a$ & $2 b$ & $2 \mathrm{c}$ & 3 & Total \\
\hline 1 & & & & 3 & & & 3 \\
\hline 2 & & & & 8 & 2 & 1 & 11 \\
\hline 3 & & & & 1 & 3 & 1 & 5 \\
\hline $4 a$ & & & & 3 & 4 & 1 & 8 \\
\hline $4 b$ & & & & 1 & 1 & 1 & 3 \\
\hline Total & & & & 16 & 10 & 4 & 30 \\
\hline
\end{tabular}


Table 3 Summary of the induction chemotherapy and concurrent chemoradiotherapy in the patients with oropharyngeal squamous cell carcinoma

\begin{tabular}{|c|c|c|c|c|c|c|c|c|c|c|c|c|c|}
\hline \multirow{2}{*}{ Case\# } & \multirow{2}{*}{ Age } & \multirow{2}{*}{ Gender } & \multirow{2}{*}{ Tumor subsite } & \multirow{2}{*}{ HPV } & \multirow{2}{*}{$\mathrm{T}$} & \multirow{2}{*}{$\mathrm{N}$} & \multirow{2}{*}{ Stage } & \multirow{2}{*}{$\begin{array}{c}\text { No. of DCF } \\
\text { cycles } \\
\text { administered }\end{array}$} & \multicolumn{2}{|c|}{$\begin{array}{c}\text { Response after } \\
\text { ICT }\end{array}$} & \multirow{2}{*}{$\begin{array}{l}\text { Total dosage } \\
\text { of cisplatin } \\
\text { during CRT } \\
\quad\left(\mathrm{mg} / \mathrm{m}^{2}\right)\end{array}$} & \multicolumn{2}{|c|}{$\begin{array}{c}\text { Response after } \\
\text { CRT }\end{array}$} \\
\hline & & & & & & & & & primary & neck & & primary & neck \\
\hline 1 & 61 & $\mathrm{M}$ & Anterior wall & HPV16 & 1 & $2 b$ & IVA & 2 & $\mathrm{CR}$ & PR & 200 & $\mathrm{CR}$ & $\mathrm{CR}$ \\
\hline 2 & 56 & $\mathrm{M}$ & Lateral wall & HPV16 & 2 & $2 b$ & IVA & 3 & PR & PR & 160 & $\mathrm{CR}$ & $\mathrm{CR}$ \\
\hline 3 & 40 & M & Lateral wall & HPV16 & 2 & $2 b$ & IVA & 1 & PR & PR & 200 & $\mathrm{CR}$ & $\mathrm{CR}$ \\
\hline 4 & 46 & $\mathrm{M}$ & Lateral wall & HPV16 & 3 & 3 & IVB & 3 & PR & PR & 200 & $\mathrm{CR}$ & $\mathrm{CR}$ \\
\hline 5 & 44 & $\mathrm{M}$ & Lateral wall & HPV18 & $4 \mathrm{~b}$ & $2 b$ & IVB & 3 & PR & PR & 200 & $\mathrm{CR}$ & PR \\
\hline 6 & 48 & $\mathrm{M}$ & Lateral wall & HPV16 & $4 \mathrm{~b}$ & $2 \mathrm{c}$ & IVB & 3 & PR & PR & 160 & $\mathrm{CR}$ & $\mathrm{CR}$ \\
\hline 7 & 69 & M & Anterior wall & negative & 1 & $2 \mathrm{~b}$ & IVA & 3 & PR & $\mathrm{SD}$ & 120 & $\mathrm{CR}$ & $\mathrm{CR}$ \\
\hline 8 & 68 & $\mathrm{M}$ & Posterior wall & negative & 3 & $2 \mathrm{c}$ & IVA & 2 & $\mathrm{CR}$ & PR & 240 & $\mathrm{CR}$ & $\mathrm{CR}$ \\
\hline 9 & 61 & $\mathrm{M}$ & Posterior wall & negative & 3 & $2 \mathrm{c}$ & IVA & 3 & $\mathrm{CR}$ & PR & 200 & $\mathrm{CR}$ & $\mathrm{CR}$ \\
\hline 10 & 57 & $\mathrm{~F}$ & Lateral wall & negative & $4 \mathrm{a}$ & $2 b$ & IVA & 3 & PR & PR & 120 & CR & $\mathrm{CR}$ \\
\hline 11 & 64 & $\mathrm{M}$ & Anterior wall & negative & $4 \mathrm{a}$ & $2 \mathrm{c}$ & IVA & 3 & PR & PR & 200 & CR & $\mathrm{CR}$ \\
\hline 12 & 64 & $\mathrm{~F}$ & Lateral wall & N/A & 1 & $2 b$ & IVA & 3 & $\mathrm{CR}$ & $\mathrm{CR}$ & 120 & $\mathrm{CR}$ & $\mathrm{CR}$ \\
\hline 13 & 57 & M & Lateral wall & N/A & 2 & $2 b$ & IVA & 3 & $\mathrm{CR}$ & PR & 120 & $\mathrm{CR}$ & $\mathrm{CR}$ \\
\hline 14 & 48 & M & Lateral wall & N/A & 3 & $2 \mathrm{c}$ & IVA & 3 & $\mathrm{CR}$ & $\mathrm{CR}$ & 200 & $\mathrm{CR}$ & $\mathrm{CR}$ \\
\hline 15 & 46 & M & Lateral wall & N/A & $4 a$ & $2 \mathrm{c}$ & IVA & 3 & PR & PR & 160 & $\mathrm{CR}$ & $\mathrm{CR}$ \\
\hline
\end{tabular}

$D C F$ docetaxel, cisplatin, and 5-fluorouracil, $I C T$ induction chemotherapy, $C R T$ chemoradiation, $N / A$ not available 
Table 4 Summary of the induction chemotherapy and concurrent chemoradiotherapy in the patients with hypopharyngeal and laryngeal carcinoma

\begin{tabular}{|c|c|c|c|c|c|c|c|c|c|c|c|c|}
\hline \multirow{2}{*}{ Case\# } & \multirow{2}{*}{ Age } & \multirow{2}{*}{ Gender } & \multirow{2}{*}{ Tumor site } & \multirow{2}{*}{$\mathrm{T}$} & \multirow{2}{*}{$\mathrm{N}$} & \multirow{2}{*}{ Stage } & \multirow{2}{*}{$\begin{array}{c}\text { No. of DCF } \\
\text { cycles } \\
\text { administered }\end{array}$} & \multicolumn{2}{|c|}{$\begin{array}{c}\text { Response after } \\
\text { ICT }\end{array}$} & \multirow{2}{*}{$\begin{array}{l}\text { Total dosage } \\
\text { of cisplatin } \\
\text { during CRT } \\
\quad\left(\mathrm{mg} / \mathrm{m}^{2}\right)\end{array}$} & \multicolumn{2}{|c|}{$\begin{array}{c}\text { Response after } \\
\text { CRT }\end{array}$} \\
\hline & & & & & & & & primary & neck & & primary & neck \\
\hline 16 & 52 & M & Hypopharynx & 2 & $2 b$ & IVA & 3 & $\mathrm{PR}$ & $\mathrm{PD}$ & 240 & $\mathrm{CR}$ & CR \\
\hline 17 & 62 & M & Hypopharynx & 2 & $2 b$ & IVA & 2 & $\mathrm{PR}$ & PR & 240 & CR & CR \\
\hline 18 & 69 & M & Hypopharynx & 2 & $2 b$ & IVA & 3 & $\mathrm{CR}$ & PR & 200 & $\mathrm{CR}$ & $\mathrm{CR}$ \\
\hline 19 & 57 & M & Hypopharynx & 2 & $2 b$ & IVA & 3 & $\mathrm{CR}$ & PR & 240 & CR & CR \\
\hline 20 & 61 & M & Hypopharynx & 2 & $2 b$ & IVA & 3 & PR & PR & 160 & $\mathrm{CR}$ & CR \\
\hline 21 & 56 & M & Hypopharynx & 2 & $2 \mathrm{c}$ & IVA & 3 & $\mathrm{PR}$ & $\mathrm{PR}$ & 200 & $\mathrm{CR}$ & $\mathrm{CR}$ \\
\hline 22 & 55 & M & Hypopharynx & 3 & $2 b$ & IVA & 3 & $\mathrm{CR}$ & PR & 240 & $\mathrm{CR}$ & $\mathrm{CR}$ \\
\hline 23 & 51 & $\mathrm{M}$ & Hypopharynx & $4 a$ & $2 b$ & IVA & 3 & PR & PR & 200 & $\mathrm{CR}$ & $\mathrm{CR}$ \\
\hline 24 & 69 & M & Hypopharynx & $4 a$ & $2 \mathrm{c}$ & IVA & 3 & PR & PR & 200 & PR & PR \\
\hline 25 & 56 & M & Hypopharynx & $4 a$ & $2 \mathrm{c}$ & IVA & 3 & PR & PR & 160 & $\mathrm{CR}$ & $\mathrm{CR}$ \\
\hline 26 & 62 & M & Hypopharynx & 2 & 3 & IVB & 3 & $\mathrm{PR}$ & $\mathrm{SD}$ & 80 & $\mathrm{CR}$ & $\mathrm{SD}$ \\
\hline 27 & 66 & M & Hypopharynx & $4 \mathrm{a}$ & 3 & IVB & 3 & PR & PR & 200 & $\mathrm{CR}$ & $\mathrm{CR}$ \\
\hline 28 & 48 & $\mathrm{~F}$ & Hypopharynx & $4 b$ & 3 & IVB & 3 & PR & PR & 200 & $\mathrm{CR}$ & $\mathrm{CR}$ \\
\hline 29 & 64 & M & Larynx & 2 & $2 \mathrm{c}$ & IVA & 1 & $\mathrm{CR}$ & PR & 240 & $\mathrm{CR}$ & $\mathrm{CR}$ \\
\hline 30 & 67 & M & Larynx & $4 a$ & $2 \mathrm{~b}$ & IVA & 3 & PR & $\mathrm{PR}$ & 160 & $\mathrm{CR}$ & $\mathrm{CR}$ \\
\hline
\end{tabular}

$D C F$ docetaxel, cisplatin, and 5-fluorouracil, ICT induction chemotherapy, CRT chemoradiation 
Table 5 Toxicity grade $\geqq 3$ due to induction chemotherapy

\begin{tabular}{lcccc}
\hline & Grade 3 & Grade 4 & Total & \%Grade3,4 \\
\hline Neutropenia & 11 & 10 & 21 & 70 \\
Febrile neutropenia & 7 & 7 & 23 \\
Thrombocytopenia & 1 & 1 & 3 \\
Diarrhea & 3 & 3 & 10 \\
Enterocolitis & 1 & 1 & 3 \\
Liver dysfunction & 3 & 3 & 10 \\
Hyponatremia & 3 & 3 & 10 \\
Hyperkalemia & 1 & 1 & 3 \\
\hline
\end{tabular}


Table 6 Toxicity grade $\geqq 3$ due to concurrent weekly cisplatin chemoradiation

\begin{tabular}{|c|c|c|c|c|}
\hline & Grade 3 & Grade 4 & Total & $\%$ Grade 3,4 \\
\hline Neutropenia & 6 & & 6 & 20 \\
\hline Febrile neutropenia & 2 & & 2 & 7 \\
\hline Anemia & 4 & & 4 & 13 \\
\hline Thrombocytopenia & 1 & & 1 & 3 \\
\hline Mucositis & 11 & & 11 & 37 \\
\hline Dermatitis & 4 & 1 & 5 & 17 \\
\hline Hyponatremia & 1 & & 1 & 3 \\
\hline
\end{tabular}


Figure 1

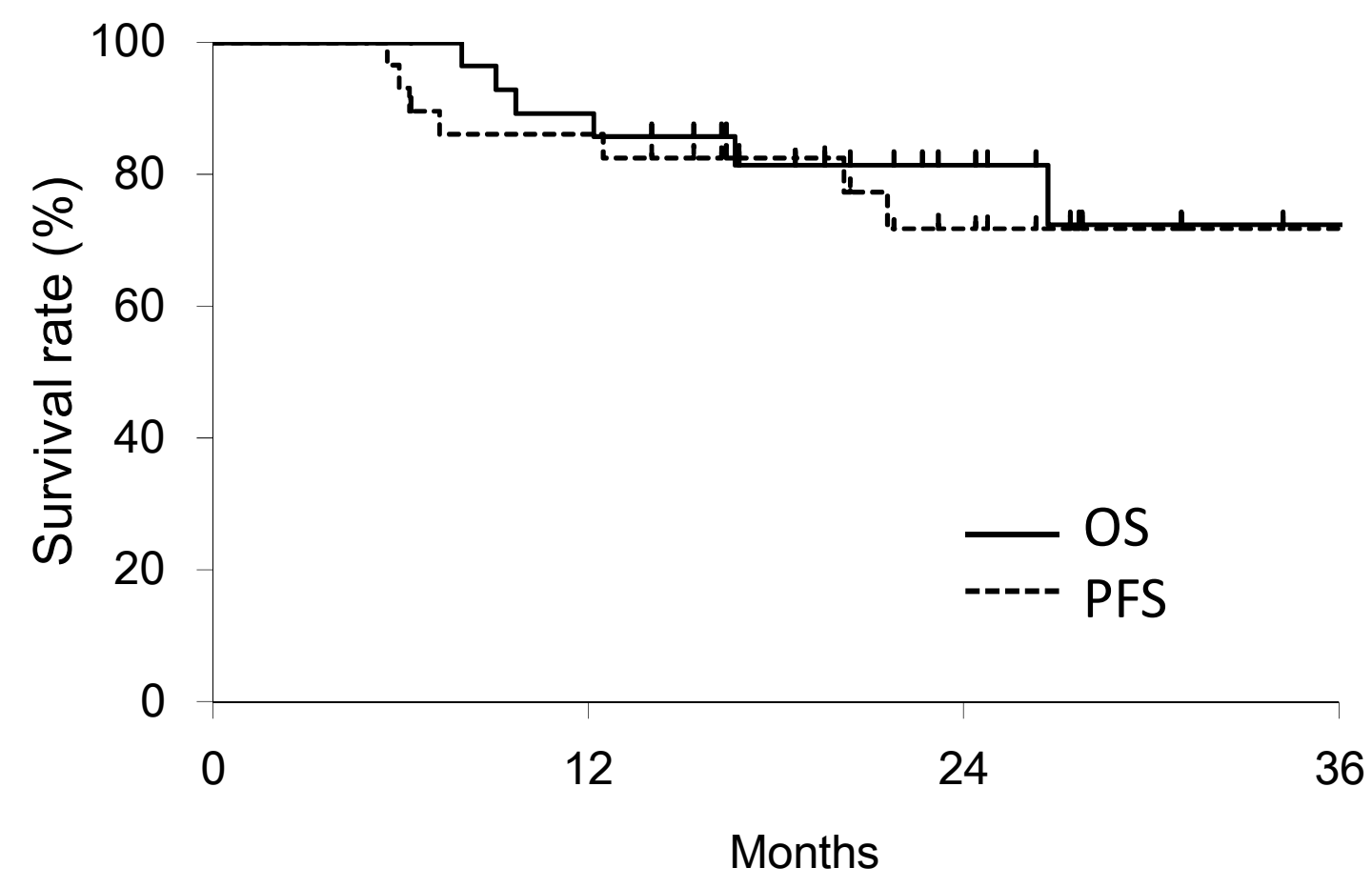

